\title{
Sistema de Gestión Universitaria de Calidad (Apreciaciones y Reflexiones)
}

\author{
University Quality Management System \\ (Appreciations y Reflections)
}

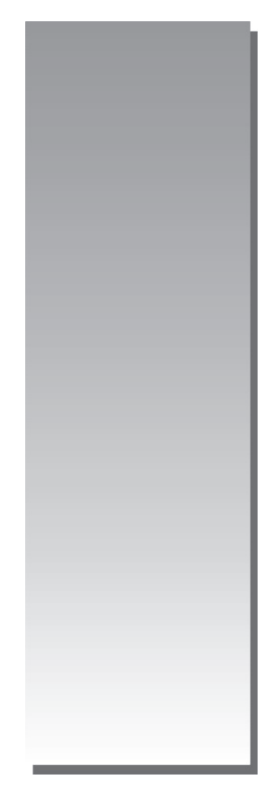

Mario Ibáñez Machicao*

Mario15022001@yahoo.com

[RECEPCIÓN: AGOSTO 2016 / CONFORMIDAD: OCTUBRE 2016]

\section{RESUMEN}

Mucho se ha hablado de la universidad peruana. En los últimos seis lustros, se dieron más de tres leyes y modificatorias o ampliatorias; todo ello, de acuerdo a intereses, influencia o presión de los poderes políticos o económicos, hasta convertir a la universidad peruana en una universidad mercantilizada. Tenemos universidad pública, privada y empresarial sin investigación en la ciencia y tecnología.

En el presente artículo, un recuento y memoria de la universidad en el mundo y la conclusión de una realidad peruana, de repente, con algunas sugerencias para un nuevo sistema educativo de calidad y un nuevo sistema de gestión universitaria de calidad para el Perú.

Palabras clave: Gestión, sistema, universidad, calidad

\section{ABSTRACT}

We talked so much about the reality of peruvian university system and, we see that in the last thirty years with more of three university laws with its complements and modifications were publishes, all of them, only for the political or economical powers of interests with the fact that, today, the peruvian university is completely merchantilized or marketing bussines. Its why, today we have public, private and managerial university. We suggest a new Peruvian educational system and, a way to orient the new peruvian educational and university quality management system.

Keywords: Management, system, university, quality 


\section{INTRODUCCIÓN}

En el Perú, por más de 190 años de vida republicana, seguimos pensando, festejando o reviviendo los legajos del pasado, no hay visión de futuro; desafortunadamente, lo pasado no nos da visión del futuro (La UNMSM se fundó el 12 de mayo de 1551, más de 460 años de historia y no figura entre las mejores universidades del mundo).

Solo una acertada gestión universitaria de calidad hará posible una educación de calidad que nos permita prever el futuro; no solamente como país, sino como universidad y como persona en los estudiantes; especialmente, los profesionales universitarios. "Como muestra basta un botón", dice el proverbio popular. China, India, Finlandia, Suecia, y especialmente Israel, en corto tiempo lograron mejorar su nivel educativo, basado en la ciencia, la tecnología y la innovación, siendo el resultado la reducción de la pobreza y, tener entre sus profesionales egresados con "Premio Nobel".

En algunas universidades peruanas, el desarrollo de la ciencia, tecnología e innovación parece no ser tarea prioritaria ni importante; claro está, no se ha aplicado una gestión universitaria de calidad.

Los peruanos seguimos pensando que la producción de bienes materiales, a base de la explotación de la materia prima, es el desarrollo de una sociedad; ello corresponde a los años 4050 del siglo pasado, donde aparecieron técnicos orientados hacia la calidad del proceso de producción: Demming, Juran y otros; (hasta en la UNI se formaban ingenieros de control de calidad; hoy, no existe). Claro, en 1950 la transformación de la materia prima era la principal actividad comercial industrial. En la actualidad, Carlos Slim, Warren Buffet, Bill Gates (los más grandes millonarios del mundo) no transforman materia prima, brindan SERVICIOS.

Estamos en la era del conocimiento, pero en el Perú casi nada se hace con nuestro conocimiento adquirido en la universidad, o es que la mayoría de nuestros egresados no recibieron una formación profesional de calidad (Nuestro vecino Venezuela, con enorme riqueza de materia prima, está con una alta tasa de pobreza por falta de una gestión universitaria de calidad; en cambio, nuestro otro vecino Chile envió a más de 5000 profesionales a seguir estudios de postgrado en el extranjero, una decisión que mira hacia el futuro.

\section{OBJETIVO DEL ARTíCULO}

Dar a conocer la realidad del sistema de gestión universitaria peruana, con relación a otras universidades del mundo con un Sistema de gestión universitaria de calidad, a manera de reflexión.

\section{ARGUMENTACIÓN}

Si en 465 años de vida universitaria no hemos hecho una universidad científica, tecnológica e innovadora, razonemos para demostrar que podemos cambiar. El artículo contiene referencias, hechos y realidades de otras universidades del mundo, que propongo como reflexión para una automotivación.

\section{HAY QUE MIRAR HACIA ADELANTE}

Todos los países lo hacen, mirando siempre el futuro, hacia un desarrollo socioeconómico de su población para mañana; en el campo del desarrollo tecnológico, tanto Europa como EE.UU. imitaron lo que Japón hizo, mediante los "círculos de calidad" y "calidad total". Los japoneses son los grandes pensadores y gestores de la calidad.

Bill Gates en una oportunidad dijo: "Lo que más me asusta de China e India es su nivel de humildad, todo lo que China hace, India lo imita y viceversa”; en el Perú, somos triunfalistas y nos gusta vivir de nuestra gloriosa historia.

En 1980, EE.UU. pensaba que Japón iba a superarlos en todo (Japón alcanzó a ser la primera potencia industrial, económica y tecnológica en el mundo) solo basado en sus famosos "círculos de calidad" y la "filosofía de la calidad total", creados por Kauro Ishikawa. En el país del norte y toda Europa aplicaron esas enseñanzas, es decir, imitaron.

EE.UU., con un sentimiento de humildad basado en la calidad de sus técnicos profesionales, hizo esfuerzo en todos los campos y recuperó su hegemonía mundial. Este es el resultado de una gestión universitaria de calidad; en consecuencia, en la universidad peruana, con humildad también, deberíamos empezar a aplicar una gestión universitaria de calidad.

Aceptemos que las universidades peruanas son las vacas sagradas, por su autonomía universitaria (muchos pensadores de la pedagogía universitaria peruana lo han confirmado así). Seguimos celebrando como grandes triunfos los 
acontecimientos estudiantiles del siglo pasado en Córdova y Cuzco (1918): "La universidad: un refugio de mediocres fiel reflejo de las sociedades decadentes". Preguntémonos: ¿Cuánto hemos cambiado? Seguimos en los últimos lugares de los rankings internacionales y con una Ley universitaria irrealista. ¿Acaso somos conformistas y aceptamos nuestra educación superior tal como es?

No hay propuestas alentadoras. La tan esperada y comentada Nueva Ley Universitaria aún no despierta.

\section{LA UNIVERSIDAD: BASE REFERENCIAL DEL DESARROLLO SOCIOECONÓMICO, TECNOLÓGICO Y CIENTÍFICO DE UN PAÍS \\ (Datos referenciales)}

Como referencia, de las 100 mejores universidades del mundo (Ranking Web): Harvard encabeza la lista, en el lugar 50 está la UNAM de México y Singapur (que tiene cerca de 6 millones de habitantes con un PIB de $\$ 225,000$ millones) ocupa ya el lugar 83 en el ranking internacional. Perú no aparece.

En las universidades peruanas más del $50 \%$ de los estudiantes cursan carreras de Derecho, Historia, Sociología, Educación, Psicología, Administración, Contabilidad; solamente el 10\% ingeniería, tecnología y ciencias. No hay carreras que incentiven la investigación y la innovación para el futuro.

En China, 1242000 estudiantes ingresan a Ingeniería, en India es caso parecido.

En la universidad peruana, no hay inversión en Investigación y desarrollo de nuevos productos y/o servicios, con rara excepción (UNALM-Lima).

Lo alarmante es que el próximo 12 de mayo vamos a cumplir 466 años de vida universitaria, las universidades se multiplican y se mercantilizan (1960: Perú con 6’000,000 de habitantes, tenía menos de 20 universidades); hoy, más de 140; sus estamentos se perennizan, todo ello por la debilidad de la ley y la inexistencia de una gestión universitaria de calidad; por ello, no hay desarrollo tecnológico, innovación ni, menos, presencia cultural en el país, como debería ser como producto de una gestión universitaria de calidad.

Si el resultado de una gestión universitaria de calidad es: formación profesional, investigación científica con proyección social y extensión universitaria, preguntémonos: ¿Cuántas patentes universitarias tenemos registradas en el mercado mundial?

En el Perú, nos encerramos en nuestro territorio, mientras que, por ejemplo, Singapur, en 2013, tenía 4000 estudiantes universitarios becados en EE.UU. Singapur, gracias a una gestión universitaria de calidad, en 30 años, pasó de un país del tercer mundo a uno de los más avanzados del primer mundo. Hoy es el mayor productor de servicios de ingeniería y arquitectura a nivel mundial.

En Taiwán, otro tigre asiático, egresan ingenieros electrónicos talentosos, cuyo resultado es: fabrican el $85 \%$ de la producción mundial de teclados para la computadora.

Como referencia del desarrollo socioeconómico de algunos países: En 1960 el PBI de Argentina y Venezuela era 10 veces mayor que el de Corea del Sur; hoy, Corea del Sur, 27,000.

En la actualidad, gracias a una gestión universitaria de calidad, Corea del Sur, con sus profesionales universitarios, tiene 7500 patentes internacionales registrados frente a 100 de Brasil y Perú: ...?

Corea del Sur, producto del fomento de la investigación universitaria, alcanzó ese liderazgo de desarrollo tecnológico de la ingeniería electrónica y la metalmecánica. En 2008, Corea del Sur registró 80,000 patentes a nivel mundial, Perú 7. En 2016, es el primer país innovador del mundo (Samsung acaba de lanzar al mercado una refrigeradora inteligente interconectada al banco y el supermercado para comprar y pagar desde la cocina de la casa).

\section{REFERENCIAS DE UNA GESTIÓN UNIVERSITARIA DE CALIDAD}

En Microsoft-USA, igualmente, gracias a una gestión empresarial de calidad de sus directivos, de los cerca de 25000 investigadores de software, 800 investigadores son generadores de patentes, de los cuales $40 \%$ son asiáticos.

(Otra referencia estadística: IBM Corp-USA. Tiene 4900 patentes, Samsung 3600 y Microsoft 2900, en el Perú...?).

Patentes: Organismo mundial de la propiedad intelectual: Ranking de número de patentes registrados, a 2016:

\section{- Japón}


- EE.UU.

- China

- Corea del Sur.

Bill Gates, en infinidad de ocasiones, expresó: "tuve una educación de muy buena calidad. En la mayor parte de otros lugares del mundo yo hubiera sido un mal agricultor". Era consciente de que había tenido la fortuna de asistir a una escuela secundaria donde se había instalado una computadora y es de suponer que la gestión administrativa de la escuela era de calidad.

EE.UU. está entre los países que más invierten en investigación universitaria y tienen una relación más estrecha con las incubadoras de nuevas empresas; las universidades otorgan incentivos financieros a sus profesores para que investiguen y comercialicen sus nuevos productos/servicios. Así, contribuye a su superior casa de estudios; por ello, EE.UU. sigue siendo el imán para los mejores cerebros de todo el mundo.

\section{LA CREATIVIDAD Y LA INNOVACIÓN VIENEN DE UN BUEN SISTEMA DE GESTIÓN UNIVERSITARIA DE CALIDAD}

En el Perú, si se estimula la curiosidad intelectual de los jóvenes por la ciencia y la tecnología (ingeniería), podemos ser un país maravilloso como Japón, Corea del Sur, Singapur o Finlandia.

Solo hay que partir desde una gestión universitaria de calidad, renaciendo a ser más cuidadoso y selectivo en los tres estamentos: Profesores de calidad, administrativos de calidad, estudiantes de calidad y, por sobre todo, autoridades de calidad.

La UNESCO resaltó algo bueno del Perú: la UNALM es la única que sobresale debido a que ha sido muy celosa en seleccionar el mejor elemento profesor y el mejor elemento estudiante, mejor laboratorio (metros cuadrados de terreno) para la investigación del estudiante.

La filosofía de la calidad total japonesa parte de la premisa de que si todos los elementos componentes de una organización social (dígase empresa o institución universitaria) son de calidad la calidad del producto final (un bien material o servicio) es consecuencia natural de la calidad de sus elementos componentes de la organización social.
En el caso de la institución universitaria, si sus elementos constitutivos: rector, vicerrector, decanos, directores, jefes, coordinadores, docentes, no docentes, estudiantes, normas (estatuto que se cumpla), equipos, herramientas, instalaciones, laboratorios, servicios, infraestructura académica, actitudes como: las decisiones, órdenes, disposiciones, acuerdos, resoluciones y demás son de calidad, el producto (el egresado) será consecuencia natural de la calidad de los elementos constitutivos de la universidad, un egresado de calidad. A ello, nos conduciría una gestión universitaria de calidad.

(En la Cumbre de las Américas 2005, los presidentes versaron sobre Bolívar, la injerencia de EE.UU. en América latina del XIX, la invasión a Bahía de Cochinos, etc. Obama, acertadamente, dijo: No he venido aquí para debatir el pasado. He venido aquí a lidiar con el futuro. El futuro del Perú tiene que tener su base en un nuevo sistema educativo, especialmente la formación universitaria, pero con un nuevo sistema de gestión universitaria de calidad.

\section{EL ESTUDIANTE UNIVERSITARIO EN EL PERÚ}

En el Perú, solo el 10\% de los jóvenes que terminan secundaria tienen acceso a la universidad (500 mil jóvenes culminan su secundaria anualmente), comparado con el $70 \%$ de ingresantes en los países industrializados. ¿No será por la mala calidad de la educación primaria y secundaria?

En la mayoría de las universidades exigentes, tanto públicas como privadas, del $100 \%$ de ingresantes, solo el $40 \%$ termina su formación profesional en el período de estudios (cinco años), siendo el mayor factor determinante, la falta de una conciencia de responsabilidad universitaria del estudiante y, por ello, la falta de dedicación al estudio y, en pocos casos, el factor económico. (Lo curioso: hay universidades privadas donde el $100 \%$ de ingresantes terminan sus estudios en los cinco años).

\section{PERÚ CON VACACIONES MÁS LARGAS DEL MUNDO}

Japón: año escolar con 243 días; Corea del Sur, con 220 días; en el Perú menos de 160 días, los restantes 205 días del año son de vacaciones para los niños y jóvenes. 
Los niños de China, India y Singapur estudian 12 horas diarias. En el Perú, un promedio de 5 horas efectivas.

La verdad es que los asiáticos viven obsesionados con el futuro; en el Perú vivimos emocionados por el pasado.

La universidad se creó con la finalidad de formar profesionales en una especialidad, la investigación científica y extensión universitaria y proyección social.

En la práctica actual, algunas universidades privadas son "fabricantes de profesionales"; frente a esta realidad, un sistema de gestión universitaria de calidad tiene que garantizar no solamente una formación profesional, sino un egresado moralmente bien formado; la universidad tiene que tender a orientar a su egresado a ser más competitivo, no solamente como persona o profesional, sino también como futuro empresario o administrador del Estado que permitiría reducir la pobreza y elevar el nivel de vida de nuestra población.

\section{LA IMAGINACIÓN Y EL CONOCIMIENTO EN LA UNIVERSIDAD}

Recordemos que el mercado de valores, Wall Street, los adelantos tecnológicos de Taiwán y Corea del Sur, el viaje al espacio, el tren Shinkansen de Japón fueron hechos realidad o creados por profesionales universitarios investigadores de calidad y, con énfasis, formados bajo un sistema de gestión universitaria de calidad.

Nosotros seguimos soñando con el pasado, bonita historia, pero Bolívar y San Martín murieron 180 años antes de que existiera el Internet, creado por ingenieros investigadores, producto de la imaginación y el conocimiento que proporciona un sistema de gestión universitaria de calidad.

En el Perú, las novelas de TV son las preferidas por la población; en EE.UU las obras como "En busca de la excelencia", "La competitividad", "Calidad total" son los "best sellers".

La China e India de hoy, está reduciendo la pobreza. ¿Cómo?: mirando al mundo para ver qué se está haciendo y copiar ese "conocimiento" de progreso para aplicarlo, Claro está, porque les conviene.

En el Perú, lo usual es mirar hacia adentro: iQué maravilloso es el Perú!, "Primero lo nuestro".
Somos uno de los países más ricos del mundo en recursos naturales, pero seguimos siendo un país atrasado, la materia prima se va agotando y achicando; además, es para la exportación, no es para el peruano (las autoridades políticas alardean: Somos el primer productor y exportador de quinua. iQué grandioso! Mientras nuestra población sufre hambre).

China e India reciben con aplausos a las universidades norteamericanas y europeas para que abran sucursales en sus territorios y compitan con las universidades locales; en el Perú, "somos nacionalistas", no aceptamos universidades extranjeras ni profesores extranjeros "injerencia extranjera”; pero sí apoyamos el nacimiento de nuevas universidades (universidades que no deberían ser universidades) (Así lo expresó el mismo Presidente de la República - 2013).

No debemos olvidar que el Perú tuvo una universidad (UNMSM) antes que EE.UU. y seguimos siendo un país atrasado debido a la no existencia de un sistema de gestión universitaria de calidad.

Cuando se dio la revolución industrial en Inglaterra, los países: Alemania, Francia, EE.UU, Canadá, Australia, siguieron esa línea de la industrialización; sin embargo, en el Perú, la revolución industrial pasó como una bala. Nada aprendimos y seguimos dependiendo de nuestra materia prima y no hemos invertido nada para una gestión universitaria de calidad en la formación profesional superior de nuestros jóvenes; claro está, con algunas excepciones (ilustres peruanos de calidad: Javier Pérez de Cuellar, Mario Vargas Llosa, José Luis Bustamante y Rivero, Ing. Carlos Noriega, Lic. Adm. Bruno Di Leo, entre otros). Podemos afirmar que la universidad peruana, especialmente en su nivel de maestría y doctorado, se ha mercantilizado.

Basta ver el local de una universidad pública limeña, donde los estudiantes de maestría y doctorado (todos trabajan), en los días de clases, sábados y domingos por las mañanas: es un hormiguero; y las autoridades se vanaglorian de tener a ese nivel más de 5000 alumnos que egresan en tiempo corto.

La interrogante sería: ¿Cuándo investigan y cuál ha sido la producción intelectual de sus graduados? Si en estos niveles superiores no hay investigación, la conclusión certera es que no hay innovación. No hay creación porque no hay producción intelectual y la gestión universitaria no es de calidad. 


\section{UNA EDUCACIÓN MIRANDO HACIA EL FUTURO}

Muchos historiadores de la educación lo han afirmado: El peor enemigo de toda sociedad en el mundo es la falta de educación. En los países en vías de desarrollo, seguimos discutiendo sobre el capitalismo y el socialismo, propios del siglo pasado; estamos en la era del conocimiento, donde las teorías económicas y tecnológicas nos orientan sobre la competitividad, la globalización de los mercados y la filosofía de la calidad total en todos los campos de la vida humana.

Los asiáticos nos están demostrando que son más pragmáticos, miran el futuro; y mirar el futuro es dedicarse hoy a la investigación y desarrollo de la ciencia y la tecnología, no hay otra alternativa. Todo ello se imparte mediante un sistema de gestión universitaria de calidad, donde la universidad sigue siendo el eje central para el desarrollo socioeconómico de un país. Como muestra basta un botón, versa un proverbio muy popular que nos trae el ejemplo de la Universidad de Harvard. Una universidad joven frente a otras que tienen el doble de vida académica y vemos a EE.UU. como el mejor país desarrollado del mundo en las ciencias y la tecnología.

En el Perú, si seguimos en este tren de vida, al que nos hemos acostumbrado en toda nuestra historia republicana, pienso que en otros 200 años el Perú no cambiará.

Para que el Perú cambie, se tiene que partir cambiando desde la raíz, y la raíz de todo elemento es la base; en este caso, la base del cambio en el Perú tiene que empezar con un nuevo sistema educativo, una educación diseñada por expertos y conocedores de la educación peruana y sus aspiraciones (no por políticos). El experto y conocedor es aquel que ha tenido experiencia laboral profesional en la educación primaria, secundaria, técnica; ha ocupado cargos ejecutivos en empresas -para conocer la realidad práctica- y, cargos directivos en universidades pública-privada, que por seguro crearán un nuevo sistema educativo (nacional o importado) para una nueva generación de peruanos, empezando desde el nivel de transición, primaria, secundaria y llegar al nivel universitario con una nueva mentalidad, con aspiraciones, con dominio del manejo de la computadora, con bases sólidas en el desarrollo científico y tecnológico. Al final, ese nuevo peruano, con una conciencia de responsabilidad nacionalista, con una nueva mentalidad en su conducta profesional, será el nuevo peruano profesional universitario formado para ser un buen ejecutivo, dirigente, empresario, investigador, creador, innovador, emprendedor y/o político visionario (no politiquero), conocedor del Perú profundo. Ese nuevo peruano será la solución a los grandes problemas socioeconómicos que aquejan al Perú, los que arrastramos a lo largo de los 196 años de vida republicana.

\section{¿QUÉ ES LO QUE DEBEMOS HACER EN EL PERÚ?}

Como ejemplos: China ha sido un país aislado del mundo; ahora, con su nueva política de internacionalización de la universidad, ya tiene cerca de 1000 programas y más de 150 universidades extranjeras. Algunas universidades estadounidenses se han trasladado con sus propios programas y hasta profesores. La Universidad de Florida tiene más de mil alumnos en Turismo y Hotelería, se trasladó con sus propios profesores y con título por la Universidad de Florida.

Tenemos que reconocer que en los EE.UU. existe un sistema de gestión universitaria de calidad; por ello, India tiene cerca de 100,000 universitarios becados allí, China igual cantidad, Corea del Sur cerca de 70,000 y nosotros, el Perú. (En cierta ocasión, INABEC dijo: Perú perdía un $30 \%$ de oferta de becas porque el postulante no sabía inglés).

\section{LO ANECDÓTICO, PARA MEDITAR}

Los países con más recursos naturales somos los países más pobres y los países con menos recursos naturales son los más ricos.

Singapur: cero de recursos naturales; Japón $75 \%$ de su territorio totalmente rocoso volcánico e improductivo; igualmente Taiwán, Honk Kong. Los tigres asiáticos tienen solo el cerebro de sus profesionales universitarios como "materia prima", pero gracias a un sistema de gestión universitaria de calidad, con sus profesionales de calidad, lograron ubicarse entre los países más desarrollados en el mundo (recordemos que Taiwán importó ingenieros electrónicos y administradores desde California, EE.UU).

Bolivia, Ecuador, Venezuela y Perú, con enormes recursos naturales, estamos en el fondo de la lista de ricos y en los primeros lugares de extrema pobreza.

Singapur, gracias al énfasis que puso para mejorar su sistema educativo, pasó de ser un país del tercer mundo a uno de los más avanzados del 
primer mundo. Hoy es el mayor exportador de plataformas petroleras submarinas y uno de los mayores productores de servicios de ingeniería y arquitectura del mundo, producto de una gestión universitaria de calidad en la formación de sus profesionales universitarios.

¿Y cómo así? Solo lograron aprovechar la imaginación, la inteligencia y el conocimiento adquirido en la universidad. Nosotros, si imitáramos a ellos, a mediano plazo podemos ser mejor que EE.UU. En la página de Google está la siguiente información: un programa de computación de Google vale cuatro veces más que el producto interno bruto (PBI) de Bolivia.

En alguna parte del mundo se dijo: "El país que no educa a su población ya no tiene futuro, gracias a su materia prima, lo cierto es que en el futuro la economía mundial estará en los productos del conocimiento y los servicios, que ya lo estamos viviendo".

Los hombres más ricos del mundo: Bill Gates, Carlos Slim, Warren Buffett y Lawrence Ellison, ninguno de ellos hizo su fortuna vendiendo materias primas; Gates hizo su fortuna vendiendo programas de computación, Slim productos de telefonía celular, Buffett con operaciones bursátiles. Ellison, creador de Oracle.

"El problema fundamental del Perú no es un problema de crecimiento económico, sino problema de educación". La mala calidad de la educación primaria, secundaria, especialmente universitaria, totalmente politizada y mercantilizada, requiere de un nuevo sistema de gestión universitaria de calidad; de lo contrario, seguiremos esperando un "milagro", el milagro de una nueva ley universitaria dentro de una nueva política educativa, en provecho de las nuevas generaciones de peruanos.

\section{UNA CRUDA REALIDAD UNIVERSITARIA PERUANA}

Solo un mínimo porcentaje de profesores universitarios fueron incorporados porque tuvieron experiencia profesional de más de cinco años en una gran empresa, poseen grado de maestría y doctorado para ganar un concurso de méritos para la docencia universitaria. Los demás son, entre otros: representantes estudiantiles y no son investigadores, familiares de las autoridades, algunos perdieron su empleo o no encontraron trabajo y, otro tanto se jubiló como trabajador dependiente.
EL DESARROLLO SOCIOECONÓMICO DE LOS PAÍSES Y EL APORTE DE UN SISTEMA DE GESTIÓN UNIVERSITARIA DE CALIDAD

\section{EJEMPLO VIVIENTE (1)- (Autobiografías):}

A la pregunta hecha a Bill Gates sobre qué pueden hacer los latinoamericanos para mejorar sus niveles educativos, como China e India, y ser más competitivos en la economía mundial, este respondió:

"La mejor manera de empezar es sintiéndose mal, con humildad".

Perú tendrá que sentirse como se siente China e India, y como se sentía EE.UU en la década de los ochenta.

En 1988, EE.UU. pensaba que Japón les superaría, debido a que en los rankings internacionales habían concluido que Japón estaba a punto de superar a EE.UU. en la carrera mundial por el liderazgo en la innovación. "EE.UU. decía: Los japoneses tienen un mejor sistema educativo, trabajan más, piensan a largo plazo".

Las grandes empresas norteamericanas se preocupaban, los japoneses van a arrasar con nosotros. Y eso fue lo que llevó a EE.UU. a ponerse a trabajar, y crear la microprocesadora, internet, Microsoft. La humildad fue un factor que ayudó mucho", añadió Gates.

\section{EJEMPLO VIVIENTE (2) - Finlandia: (5 millones de habitantes)}

\section{¿Qué ha hecho este país?}

Finlandia hasta hace pocas décadas vivía de la agricultura y de la exportación de sus materias primas.

Para llegar a los primeros puestos de los más importantes rankings internacionales que miden el éxito socio-económico-político de las naciones.

Finlandia, el país más pobre de Europa, figura en los primeros lugares del ranking de competitividad internacional, es el país más democrático, menos corrupto, los estudiantes de 15 años tienen mejor conocimiento en matemática, ciencias y lenguaje; en la universidad, mayor número de investigadores científicos.

Finlandia tiene un excelente sistema educativo: primaria, secundaria y universitaria, gratuito, con un maestro por cada 12 alumnos. 
El 75\% de las mujeres son maestras y pertenecen al estatus social elevado, con buen sueldo, trabajan cinco a seis horas diarias. Los alumnos de bajo rendimiento tienen una maestra con grado de doctora para tutoría y clases personalizadas, y mucha experiencia profesional, exclusiva para la recuperación o tutoría de los desaprobados.

En Finlandia, la idea es que ningún niño se quede atrás. El 100\% de alumnos de primaria tienen PC en contacto directo con los padres, alumno y maestra.

Los alumnos regulares con puntaje 7.5 (sobre 10) no pueden seguir estudios universitarios, sino profesiones técnicas, de tal modo que todo el mundo trata de tener un puntaje superior a 7.5.

La universidad es una de las columnas vertebrales del país. Los profesores ganan un promedio de 100,000 dólares anuales, son evaluados cada tres años por una comisión de ocho auditores encabezada por un extranjero especialista en la materia que dicta. Cada vez se dictan más cursos en inglés, $25 \%$ de docentes son de habla inglesa.

La asociación de estudiantes "Tercio estudiantil" tiene un presupuesto anual de 4,000,000 de euros, administra 200 millones de euros en bienes: cafetería, servicio (cada alumno recibe 3,600 dólares anuales para estudiar), el tercio publica libros como "Una nueva mente", de Daniel H. Pink, best-seller, para todo alumno. Indudablemente, ello ha sido posible gracias a un sistema de gestión universitaria de calidad.

Finlandia invierte el 3.5\% del PIB en investigación y desarrollo de nuevos productos, instituyendo que en el futuro los productos de la economía del conocimiento valdrían mucho más que el producto transformado de la materia prima.

Finlandia, de explotador de su madera (materia prima) y fabricante de muebles, se convirtió en una potencia mundial en innovación y el mayor productor de telefonía móvil en el mundo con NOKIA (Nokia 2010).

La clave del éxito finlandés está en su sistema educativo (un decreto del arzobispo luterano del siglo XVII decía: "Ningún hombre que no supiera leer podría casarse").

\section{EJEMPLO VIVIENTE (3) - SINGAPUR}

En Singapur, los billetes ilustran y muestran un grupo de jóvenes estudiantes, resaltando la importancia de la educación para la construcción del futuro.

Las tiendas comerciales tienen bibliotecas abiertas para el público (modelo japonés).

Han creado una nueva actividad docente, "Escuela de tutoría privada", donde los padres de familia llevan a sus hijos después de las clases hasta las 22:00 horas para mejorar su rendimiento académico.

La educación es la obsesión nacional y les ha permitido pasar del tercer mundo al primero en apenas cuatro décadas.

En 1965 se independizó de Inglaterra.

(Anécdota popular: "Inglaterra se deshizo de Singapur porque era una carga pesada para su economía, nadie quería adoptarla).

Hace 40 años, su PBI era menos de la mitad de Argentina (Argentina potencia sudamericana de entonces). Hoy en día, gracias a su sistema de gestión universitaria de calidad, Singapur tiene un ingreso per cápita de 520,000 dólares por año, el noveno más alto del mundo, por encima de los 47,000 de EE.UU.

La Universidad de Singapur ocupa el lugar 30 del mundo. Cuando se independizó tenía alto nivel de analfabetismo. Hoy todos los jóvenes ingresan a la universidad o institutos tecnológicos vocacionales.

Singapur se dio el privilegio de importar más de 100 universidades extranjeras, entre las mejores del mundo; ya sean como sucursales o compartido. El 20\% de sus estudiantes son extranjeros asiáticos en los 66 programas de titulación doble.

La oficina estatal de ciencia, tecnología e investigación tiene 23,000 patentes solicitadas y más de 9,800 registradas, la mayoría de las patentes en colaboración con la Universidad Nacional de Singapur.

Singapur, una pequeña isla sin recursos naturales, exporta por más de 235,000 millones de dólares. Importaba agua de Malasia hasta que lo sustituyó con la desalinización. Importaba arena. Su vecino Indonesia prohibió la exportación de arena a Singapur para que la isla no aumentara su territorio.

\section{EJEMLO VIVIENTE (4) - CHILE}

Nuestro vecino principal, los chilenos, proyectan para el futuro estar entre los primeros en el 
mundo y ser el primero de América Latina, según su reciente presidente Piñera.

Más de 6,000 profesionales becados a seguir estudios de posgrado de maestría y doctorado, ejemplar medida educativa.

Hace mucho tiempo un grupo de investigadores chilenos visitaron Noruega, exclusivamente para aprender la crianza de salmones; ahora, los chilenos son grandes exportadores de este producto de agua dulce.

El Gobierno peruano nos debería llevar a estas realidades como una necesidad de mejorar el nivel educativo superior, mediante un sistema de gestión universitaria de calidad, donde los mejores egresados, profesionales de calidad, sean becados al extranjero y, mañana, de repente, estaríamos exportando... agua.

\section{PARADIGMAS HISTÓRICOS DE GESTIÓN UNIVERSITARIA DE CALIDAD \\ UNIVERSIDAD DE HARVARD, EE.UU.}

"La universidad de Harvard fue fundada en 1636, es la institución de educación superior más antigua de los EE.UU. Entre sus exalumnos tienen:

- Ocho presidentes de los Estados Unidos, entre ellos: John Adams, John Quincy Adams, Theodore y Franklin D. Roosevelt, Rutherford B. Hayes, John Fitzgerald Kennedy, George W. Bush y Barack Obama.

- Su cuerpo docente ha sido conformado por más de 40 premios Nobel.

- La universidad de Harvard cuenta con programas en las siguientes divisiones académicas: Escuelas de Artes y Ciencias, Negocios, Medicina, Derecho, diseño, Teología, Deontología, Salud Pública, Educación y el Colegio John F. Kennedy.

- A su distinguida calidad formativa-investigativa se suma una excelente infraestructura, sobresaliendo su biblioteca, la cual cuenta con más de 15 millones de volúmenes, una de las "megabibliotecas" del mundo.

- Harvard, además, cuenta con un amplio programa cultural, a través del cual ofrece a sus alumnos cursos de danza, música, cine, artes manuales, teatro, entre otros.

- Otro de sus atractivos es el plan deportivo, que incluye la práctica de casi todos los deportes.
- Entre el año 2004 y 2009, ingresaron a Harvard 22,903 estudiantes internacionales; 3207 de ellos de origen latinoamericano: México con 488, Brasil con 407, Argentina 236 y Colombia con 207".

\section{INSTITUTO TECNOLÓGICO DE CALIFORNIA-EE.UU.}

"El Instituto Tecnológico de California (Caltech) ha sido fundado en 1891 con la misión de expandir el conocimiento en beneficio de la sociedad, integrando la investigación a la educación.

Manteniendo su propósito inicial, este instituto recibe cada año cerca de dos mil alumnos para cursar sus estudios de pregrado y posgrado; oferta académica enfocada en seis áreas de estudio: Biología, Química e Ingeniería Química, Ingeniería y Ciencias Aplicadas, Geología y Ciencias Planetarias, Humanidades y Ciencias Sociales; y Física, Matemáticas y Astronomía.

A la fecha, más de 20000 estudiantes se han graduado de Caltech, entre los cuales se encuentran:

- -17 científicos ganadores del Premio Nobel;

- Figuras como Frank Capra, director de cine;

- Moshe Arens, exministro israelí de defensa y relaciones exteriores;

- Chester F. Carlson, inventor de la xerografía (fotocopiado);

- Arnold O. Beckman, fundador y presidente emérito de Beckman Instruments;

- Steingrímur Hermannsson, primer exministro de Islandia; entre otros.

Caltech brinda a sus estudiantes servicios, entre los cuales se destaca el sistema de bibliotecas, compuesto de extensos catálogos bibliográficos, recursos hemerográficos, impresos y en línea; además de mapas, informes y otros tipos de documentación.

Sustentando su sistema educativo investigativo, Caltech cuenta con una amplia gama de laboratorios y centros de investigación enfocados en sus respectivas áreas de estudio; como el Spitzer Science Center (SSC), centro de investigación y análisis del telescopio Spitzer de la NASA, situado en el campus de Caltech". 


\section{INSTITUTO TECNOLÓGICO DE MASSACHUSETTS (MIT) - EE.UU.}

"El MIT fue fundado por el científico William Barton Rogers en 1861, con el fin de brindar un aporte a la educación científica y técnica; sin embargo, fue en 1865 cuando se admitieron los primeros estudiantes.

William Barton impulsó el desarrollo del laboratorio de enseñanza. Especializado en ciencia y tecnología, este centro educativo, ubicado en Boston, cobija cerca de 10,000 estudiantes formados en seis grandes escuelas: Arquitectura y Urbanismo, Ingeniería, Administración, Ciencias, Humanidades, Artes y Ciencias Sociales, y la Escuela Whitaker de Ciencias de las Salud y Tecnología.

Son aproximadamente 111 mil graduados del MIT, entre los cuales se encuentran:

- Noam Chomsky, lingüista;

- Buzz Aldrin, astronauta del Apolo 11;

- Kofi Annan, exsecretario General de la ONU;

- Eugenio Garza Sada, fundador del Tecnológico de Monterrey;

- Benjamín Netanyahu, Primer ministro de Israel;

- Algunos ganadores del Premio Nobel como Richard Phillips Feynman, quien obtuvo este reconocimiento en el campo de la Física.

En su campus de 168 hectáreas y gracias a los convenios con otras universidades, Gobiernos y empresas, en el MIT se genera una interesante combinación de nacionalidades, ideas y metodologías a la contribución de una mejor educación".

\section{UNIVERSIDAD DE STANFORD EE.UU.}

"Ubicada en la zona de Palo Alto, California, esta superior casa de estudios se estableció en honor al hijo del fundador, Leland Stanford Jr.

En su inauguración ingresaron 559 estudiantes; desde entonces, la universidad ha ido creciendo numéricamente en cuanto a estudiantes y colaboradores.

El campus, ubicado en lo que fuera antes la granja de Leland Stanford, está compuesto de siete edificios, en los cuales se ofrecen programas académicos en siete facultades: Ciencias de la Tierra, Ingeniería, Humanidades, Negocios, Educación, Derecho y Medicina.

En contribución con sus políticas institucionales y de comunicación, cada facultad cuenta con un periódico en línea donde se publican las novedades sobre su campo de estudio y las noticias que se generan en el mismo.

La comunidad universitaria se ha constituido de importantes personalidades, entre las que se encuentran:

- 27 ganadores del Premio Nobel;

- Se han gestado proyectos como Sun, Microsystems, Google, Yahoo, HewlettPackard y Cisco Systems".

\section{UNIVERSIDAD DE PRINCETON - EE.UU.}

"La Universidad de Princeton está ubicada en el Estado de New Jersey, es la cuarta más antigua de los EE.UU. Desde su fundación en 1748, ha sido una de las universidades más importantes de EE.UU. Esta institución universitaria ofrece programas académicos vinculados a cuatro campos de estudio: Humanidades, Ciencias Sociales, Ciencias Naturales e Ingeniería.

- En sus aulas se han formado profesionales de 98 países del mundo.

- Entre los cuales han sobresalido 14 Ganadores del Premio Nobel.

- 18 de sus administrativos y docentes han sido premiados con este galardón.

- Recibe cada año aproximadamente 6000 estudiantes en sus programas de pregrado y posgrado.

- Tiene aproximadamente 12000 trabajadores para su funcionamiento.

- La sede central tiene 180 edificios en 500 hectáreas.

- En el área residencial del campus vive el 98\% de los estudiantes universitarios.

En cuanto a su programa extracurricular, Princeton ha diseñado una amplia gama de actividades sociales, pedagógicas, culturales y deportivas. Cada una de ellas está diseñada para descubrir talentos y promover el respeto por las distintas formas de expresión, pilar de esta comunidad pluralista". 


\section{UNIVERSIDAD DE CAMBRIDGE - REINO UNIDO}

"La Universidad de Cambridge es uno de los centros superiores anglosajones más reconocidos del mundo y una de las más antiguas del Reino Unido. Su origen se remonta al período medieval, cuando fue fundada, según cuenta la historia, por algunos académicos que huyeron de la Universidad de Oxford, en 1209.

La Universidad de Cambridge cuenta con 31 universidades adscritas, de las cuales tres son únicamente para mujeres y cuatro solo para estudiantes de posgrado.

Cuenta con más de 150 departamentos, facultades, escuelas y otras instituciones.

En sus aulas han cursado estudios grandes personalidades, como científicos, políticos y escritores, entre los cuales se destacan:

- Isaac Newton -Ley de la gravitación del Universo,

- Charles Darwin, Teoría de la evolución,

- John Milton - Paraíso perdido

- y otros.

Igualmente, han sido miembros de la Universidad de Cambridge, y sus instituciones adscritas, distinguidos ganadores del Premio Nobel como:

- Bertrand Russell, Literatura;

- Dorothy Hodgkin, Química, primera mujer de la universidad en obtener este premio.

- Joseph Stiglitz en Economía.

El departamento de prensa de la Universidad de Cambridge publica los mejores escritos educativos y académicos de todo el mundo y está dedicado a fomentar el conocimiento, la educación, el aprendizaje y la investigación.

Desde su primera publicación en 1584 , la universidad imprime más de 2500 títulos y 200 revistas al año".

\section{UNIVERSIDAD DE OXFORD, REINO UNIDO}

"La Universidad de Oxford continúa siendo una de las mejores instituciones de educación superior en Reino Unido, de acuerdo con el reporte anual de la organización “Times Good University Guide”.
Uno de los aspectos que la distingue es el sistema tutorial que ofrece a los estudiantes de pregrado, ya que los estudiantes tienen la oportunidad de reunirse regularmente con un tutor en grupos de máximo dos personas. De acuerdo con el estudio presentado por el "Financial Times Global Rankings" en enero de 2010:

- El programa de Said Business School, MBA, se clasificó como 16 en el mundo y el segundo en el Reino Unido.

- La escuela de negocios también se destacó dentro de las 20 mejores en Europa (diciembre de 2009) y las 20 mejores en el mundo (enero 2009).

- Según estadísticas de Oxford, el 93\% de los estudiantes que se graduaron en verano de 2009 se vincularon a la vida laboral seis meses después de graduarse.

- En los períodos 2008-2009, Oxford invirtió el 31\% de sus ingresos adicionales por comisiones, en becas para los estudiantes de menores ingresos, en comparación con un promedio general de $25,8 \%$ en el resto de instituciones universitarias".

\section{UNIVERSIDAD DE CALIFORNIA BERKELEY - EE.UU.}

"La Universidad de California Berkeley tiene una amplia oferta académica distribuida en 14 facultades y escuelas. Dentro de esa categoría destacan sus programas de doctorado, los que la ubican dentro de las 10 mejores universidades de los Estados Unidos en ese campo, de acuerdo con un estudio elaborado por el Consejo Nacional de Investigación en setiembre de 2010. Para ingresar a Berkeley, los postulantes cuentan con varias opciones de financiamiento, entre las cuales están: estudiar y trabajar en la universidad y créditos en sus pensiones de enseñanza; además, cuentan con becas de estudios gestionadas desde la universidad y su conexión con algunas asociaciones.

La Universidad de California Berkeley ha desarrollado un extenso programa de investigación, organizado en más de 80 unidades de investigación interdisciplinaria; este proceso involucra a estudiantes y docentes, quienes recogen sus percepciones y comparten sus resultados a través de publicaciones como "Berkeley Science Review", el "Forefront" y el "Research at Berkeley". 


\section{INSTITUTO IMPERIAL DE LONDRES, REINO UNIDO}

"El Instituto Imperial de Londres permanece en la lista de las mejores del Reino Unido y ocupa el tercer lugar de las mejores universidades europeas, de acuerdo con un estudio realizado por Times Higher Education. Fundada en 1907, esta superior casa de estudios ha mantenido un promedio de más de 13 mil estudiantes, durante los períodos de 2008-2009, provenientes de 158 países.

Para los alumnos ingresantes se ofrece una amplia oferta de becas, algunas en convenio con organizaciones privadas, como la firmada con el Grupo Santander de España, que brinda una oportunidad a los estudiantes de la Península Ibérica y de América Latina.

El Instituto Imperial de Londres, especializado en ciencia, medicina y negocios, obtiene su mayor reconocimiento y prestigio en ingeniería y tecnología.

La formación académica ofrecida atrae a los gobiernos de diferentes países para formar sus profesionales, empresas privadas y medios de comunicación para educar a sus trabajadores.

Dentro de sus principales propósitos de formación profesional, el Instituto Imperial de Londres prioriza en sus estudiantes la investigación, cuyos estudios son publicados no solo en los medios de la universidad, sino en los principales diarios del Reino Unido y el mundo.

Para esta actividad, la institución cuenta con varios organismos adscritos como el Instituto Granthan para el Cambio Climático y el Laboratorio de Energía del futuro".

\section{UNIVERSIDAD DE YALE - EE.UU.}

"Abraham Pierson fundó en 1701 la primera institución universitaria estatal de Connecticut, la Escuela Collegiate, 15 años después en Killingworthy, gracias al generoso benefactor Elihu Yale, la institución no solo se trasladó a New Haven, sino que también cambió de nombre, en honor a este.

Actualmente, la universidad acoge en sus 12 edificios a aproximadamente 11000 estudiantes.

Académicamente, está compuesta por una escuela para estudiantes de pregrado, una de posgrado de artes y ciencias y 11 colegios profesionales.
Yale se distingue por ser en los EE.UU. una de las mejores universidades en el campo de investigaciones científicas y en el área de las Ciencias Sociales y Humanidades.

Los departamentos de Biología, Química, Biofísica Molecular y Bioquímica, Física, Astronomía, Matemática, Ciencias de Sistemas, Geología y otros más, también sobresalen en los rankings nacionales de educación en los EE.UU.

La universidad, además, cuenta con más de 600 programas en estudios internacionales y se enseñan más de 50 lenguas extranjeras.

Por cuatro décadas, el Centro de Estudios Internacionales Mac Millan ha sido un punto focal y, actualmente ofrece seis especialidades de licenciatura y cuatro programas de maestría, así como numerosas actividades de investigación.

Otro de los atractivos de Yale es su programa de ayuda financiera para estudiantes internacionales que sigue los mismos requisitos exigidos a los estudiantes con ciudadanía estadounidense. Los estudiantes internacionales constituyen un $16 \%$ de la población estudiantil en Yale”.

\section{REFLEXIONES}

1. A pesar de tener la universidad más antigua de América.

2. A pesar de los 466 años de vida universitaria.

3. A pesar de los 196 años de vida republicana.

4. A pesar de ser descendientes de grandes culturas como los Incas.

5. A pesar de tener 144 universidades, de todo nivel, categoría, modalidad, estatal, privada y universidad-empresa.

6. A pesar de 97 años de existencia del tercio estudiantil como cogobierno de la universidad.

7. A pesar de ser el territorio geográfico más rico del mundo.

8. A pesar de tener la flora más variada del mundo,

9. A pesar de tener la fauna más variada del mundo.

10. A pesar de poseer más del $90 \%$ de los minerales habidos sobre el globo terrestre. 
11. A pesar de tener los microclimas más ricos del mundo.

12. A pesar de tener selva extensa, valles templados, los glaciales tropicales, altiplano productivo y costa marítima más extensa.

13. A pesar de tener extensas tierras fértiles.

14. A pesar de tener la costa marítima con todas las variedades de productos marinos.

15. A pesar de tener el lago navegable más alto del mundo.

16. A pesar de tener el mayor número de lagos y ríos del mundo.

17. A pesar de haber tenido una Miss Universo y Miss Mundo.

18. A pesar de tener un Premio Nobel.

19. A pesar de tener el primer astronauta latino.

20. A pesar de ser el décimo destino turístico del mundo.

21. A pesar de tener la séptima maravilla del mundo.

22. A pesar de tener el primer aviador en cruzar el Atlántico.

23. A pesar de tener el primer latino campeón de Tenis de Wimbledon.

24. A pesar de tener el primer latino como Secretario General de las Naciones Unidas.

25. A pesar de tener el primer latino como Presidente de la Corte Superior de La Haya.

26. A pesar de ser Subcampeón Olímpico de Vóley.

27. A pesar de tener la Ciudad de los Virreyes.

28. A pesar de haber sido sede del campeonato mundial de baloncesto.
29. A pesar de haber dado al mundo el alimento básico como la papa, además del maíz y la quinua.

30. A pesar de ser un país descendiente de más de 20 culturas prehispánicas.

\section{CONCLUSIÓN.}

¿Qué es lo que está pasando con nuestro sistema educativo peruano, especialmente en el sistema de gestión universitaria que nos preocupa?

Usted, amigo lector, seguramente tiene su punto de vista, sus ideas y pareceres, en lo que sí creo que estaremos de acuerdo es que el Perú necesita, urgente y prioritariamente, un nuevo sistema educativo, para una nueva generación de profesores peruanos que se formen bajo un nuevo horizonte educativo, desde la transición, kindergarten, primaria, secundaria, profesiones técnicas y especialmente los estudios superiores universitarios, con una nueva mentalidad nacionalista progresista, investigador, creativo, innovador $\mathrm{y}$, por sobre todo, moral; diferente al sistema en el cual fuimos educados y formados. El nuevo sistema que se plasme debe ser una nueva Ley de Educación, reglamentada con una visión de futuro, para nuestros hijos, nietos y bisnietos, los futuros líderes empresariales, políticos y gobernantes del Perú; de esta forma, acabar con estos 196 años de vida republicana de inseguridad, corrupción, analfabetismo, mala salud, ausencia de trabajo, diferencia de clases, desnutrición infantil, educación mercantilizada.

\section{BIBLIOGRAFÍA}

Constitución Política del Estado. Ley General de Educación 28044 y 23384; Ley universitaria 30220,23633 , 25762, 26327 y 26439 Lima, enero, 2017. 